\title{
A Characterization Theorem in Magneto-Thermal Convection in Rivlin-Ericksen Viscoelastic Fluid
}

\author{
Ajaib S. Banyal ${ }^{1}$, Daleep K. Sharma ${ }^{2}$ \\ ${ }^{1 *}$ Department of Mathematics, Govt. College Nadaun, Dist. Hamirpur, (HP) INDIA 177033 \\ ${ }^{2}$ Department of Mathematics, Rajiv Gandhi G. C. Kotshera, Shimla (HP), INDIA 171004
}

\begin{abstract}
A layer of Rivlin-Ericksen viscoelastic fluid heated from below is considered in the presence of uniform vertical magnetic field. Following the linearized stability theory and normal mode analysis, the paper mathematically established the condition for characterizing the oscillatory motions which may be neutral or unstable, for any combination of perfectly conducting, free and rigid boundaries at the top and bottom of the fluid. It is established that all non-decaying slow motions starting from rest, in a Rivlin-Ericksen viscoelastic fluid of infinite horizontal extension and finite vertical depth, which is acted upon by uniform vertical magnetic field opposite to gravity and a constant vertical adverse free and rigid boundaries and the exact solutions of the problem investigated in closed form, are not obtainable. temperature gradient, are necessarily non-oscillatory, in the regime
\end{abstract}

$$
\frac{Q p_{2} \pi^{2}}{\left(2 \pi^{2}-1\right)\left(1+\pi^{2} F\right)} \leq 1
$$

where $Q$ is the Chandrasekhar number, $F$ is the viscoelasticity parameter and $p_{2}$ is the magnetic Prandtl number. The result is important since it hold for all wave numbers and for any combination of perfectly conducting dynamically

KeyWords: Thermal convection; Rivlin-Ericksen Fluid; Magnetic field; PES; Rayleigh number; Chandrasekhar number.

MSC 2000 No.: 76A05, 76E06, 76E15; 76E07; 76U05.

\section{Introduction}

Stability of a dynamical system is closest to real life, in the sense that realization of a dynamical system depends upon its stability. Right from the conceptualizations of turbulence, instability of fluid flows is being regarded at its root. The thermal instability of a fluid layer with maintained adverse temperature gradient by heating the underside plays an important role in Geophysics, interiors of the Earth, Oceanography and Atmospheric Physics, and has been investigated by several authors (e.g., Bénard [1], Rayleigh [2], Jeffreys [3]) under different conditions. A detailed account of the theoretical and experimental study of the onset of Bénard Convection in Newtonian fluids, under varying assumptions of hydrodynamics and hydromagnetics, has been given by Chandrasekhar [4]. The use of Boussinesq approximation has been made throughout, which states that the density changes are disregarded in all other terms in the equation of motion except the external force term. There are many elastic-viscous fluids that cannot be characterized by Maxwell's constitutive relations or Oldroyd's [5] constitutive relations. Two such classes of fluids are Rivlin-Ericksen's and Walter's (model B') fluids. Rivlin-Ericksen [6] have proposed a theoretical model for such one class of elastic-viscous fluids.

Bhatia and Steiner[7] have considered the effect of uniform rotation on the thermal instability of a viscoelastic (Maxwell) fluid and found that rotation has a destabilizing influence in contrast to the stabilizing effect on Newtonian fluid. The thermal instability of a Maxwell fluid in hydromagnetics has been studied by Bhatia and Steiner [8]. They have found that the magnetic field stabilizes a viscoelastic (Maxwell) fluid just as the Newtonian fluid. Sharma[9] has studied the thermal instability of a layer of viscoelastic (Oldroydian) fluid acted upon by a uniform rotation and found that rotation has destabilizing as well as stabilizing effects under certain conditions in contrast to that of a Maxwell fluid where it has a destabilizing effect. In another study Sharma [10] has studied the stability of a layer of an electrically conducting Oldroyd fluid [5] in the presence of magnetic field and has found that the magnetic field has a stabilizing influence.

Sharma and kumar[11] have studied the effect of rotation on thermal instability in Rivlin-Ericksen elastico-viscous fluid and found that rotation has a stabilizing effect and introduces oscillatory modes in the 
system. Kumar et al. [12] considered effect of rotation and magnetic field on Rivlin-Ericksen elastico-viscous fluid and found that rotation has stabilizing effect where as magnetic field has both stabilizing and destabilizing effects. A layer of such fluid heated from below or under the action of magnetic field or rotation or both may find applications in geophysics, interior of the Earth, Oceanography, and the atmospheric physics.

Pellow and Southwell[13] proved the validity of PES for the classical Rayleigh-Bénard convection problem. Banerjee et al [14] gave a new scheme for combining the governing equations of thermohaline convection, which is shown to lead to the bounds for the complex growth rate of the arbitrary oscillatory perturbations, neutral or unstable for all combinations of dynamically rigid or free boundaries and, Banerjee and Banerjee [15] established a criterion on characterization of non-oscillatory motions in hydrodynamics which was further extended by Gupta et al. [16]. However no such result existed for non-Newtonian fluid configurations, in general and in particular, for Rivlin-Ericksen viscoelastic fluid configurations. Banyal [17] have characterized the non-oscillatory motions in couple-stress fluid.

Keeping in mind the importance of Rivlin-Ericksen viscoelastic fluids and magnetic field, as stated above, this article attempts to study Rivlin-Ericksen viscoelastic fluid heated from below in the presence of uniform magnetic field, with more realistic boundaries and it has been established that the onset of instability in a Rivlin-Ericksen viscoelastic fluid heated from below, in the presence of uniform vertical magnetic field, cannot manifest itself as oscillatory motions of growing amplitude if the Chandrasekhar number $Q$, the viscoelasticity parameter $\mathrm{F}$ and $p_{2}$ the magnetic Prandtl number, satisfy the inequality, $\frac{Q p_{2} \pi^{2}}{\left(2 \pi^{2}-1\right)\left(1+\pi^{2} F\right)} \leq 1$, for all wave numbers and for any combination of perfectly conducting dynamically free and rigid boundaries.

\section{Formulation Of The Problem And Perturbation Equations}

Considered an infinite, horizontal, incompressible electrically conducting Rivlin-Ericksen viscoelastic fluid layer, of thickness $\mathrm{d}$, heated from below so that, the temperature and density at the bottom surface $\mathrm{z}=0$ are $T_{0}$ and $\rho_{0}$ and at the upper surface $\mathrm{z}=\mathrm{d}$ are $T_{d}$ and $\rho_{d}$ respectively, and that a uniform adverse temperature gradient $\beta\left(=\left|\frac{d T}{d z}\right|\right)$ is maintained. The fluid is acted upon by a uniform vertical magnetic field $\vec{H}(0,0, H)$, parallel to the force field of gravity $\vec{g}(0,0,-g)$.

The equation of motion, continuity, heat conduction, and Maxwells equations governing the flow of RivlinEricksen viscoelastic fluid in the presence of magnetic field(Rivlin and Ericksen [6]; Chandrasekhar [4] and Kumar et al[12]) are

$$
\begin{gathered}
\frac{\partial \vec{q}}{\partial t}+(\vec{q} \cdot \nabla) \vec{q}=-\nabla\left(\frac{p}{\rho_{o}}\right)+\vec{g}\left(1+\frac{\delta \rho}{\rho_{0}}\right)+\left(v+v^{\prime} \frac{\partial}{\partial t}\right) \nabla^{2} \vec{q}+\frac{\mu_{e}}{4 \pi \rho_{o}}(\nabla \times \vec{H}) \times \vec{H} \\
\nabla \cdot \vec{q}=0 \\
\frac{\partial T}{\partial t}+(\vec{q} \cdot \nabla) T=\kappa \nabla^{2} T \\
\nabla \cdot \vec{H}=0 \\
\frac{\partial \vec{H}}{\partial t}=(\vec{H} \cdot \nabla) \vec{q}+\eta \nabla^{2} \vec{H}
\end{gathered}
$$


Where $\rho, \mathrm{p}, \mathrm{T}, v, v^{\prime}$ and $\vec{q}(u, v, w)$ denote respectively the density, pressure, temperature, kinematic viscosity, kinematic viscoelasticity and velocity of the fluid, respectively and $\vec{r}(x, y, z)$.

The equation of state for the fluid is

$$
\rho=\rho_{0}\left[1-\alpha\left(T-T_{0}\right)\right]
$$

Where the suffix zero refer to the values at the reference level $\mathrm{z}=0$. Here $\vec{g}(0,0,-g)$ is acceleration due to gravity and $\alpha$ is the coefficient of thermal expansion. In writing the equation (1), we made use of the Boussinesq approximation, which states that the density variations are ignored in all terms in the equation of motion except the external force term. The magnetic permeability $\mu_{e}$, thermal diffusivity $\kappa$, and electrical resistivity $\eta$, are all assumed to be constant.

The initial state is one in which the velocity, density, pressure, and temperature at any point in the fluid are, respectively, given by

$$
\vec{q}=(0,0,0), \rho=\rho(z), \mathrm{p}=\mathrm{p}(\mathrm{z}), \mathrm{T}=\mathrm{T}(\mathrm{z}),
$$

Assume small perturbations around the basic solution and let $\delta \rho, \delta p, \theta, \vec{q}(u, v, w)$ and $\vec{h}=\left(h_{x}, h_{y}, h_{z}\right) \quad$ denote respectively the perturbations in density $\rho$, pressure $\mathrm{p}$, temperature $\mathrm{T}$, velocity $\vec{q}(0,0,0)$ and the magnetic field $\vec{H}=(0,0, H)$. The change in density $\delta \rho$, caused mainly by the perturbation $\theta$ in temperature, is given by

$$
\rho+\delta \rho=\rho_{0}\left[1-\alpha\left(T+\theta-T_{0}\right)\right]=\rho-\alpha \rho_{0} \theta, \text { i.e. } \delta \rho=-\alpha \rho_{0} \theta
$$

Then the linearized perturbation equations are

$$
\begin{gathered}
\frac{\partial \vec{q}}{\partial t}=-\frac{1}{\rho_{0}} \nabla \delta p-\vec{g} \alpha \theta+\left(v+v^{\prime} \frac{\partial}{\partial t}\right) \nabla^{2} \vec{q}+\frac{\mu_{e}}{4 \pi \rho_{0}}(\nabla \times \vec{h}) \times \vec{H}, \\
\nabla \cdot \vec{q}=0 \\
\frac{\partial \theta}{\partial t}=\beta w+\kappa \nabla^{2} \theta, \\
\nabla \cdot \vec{h}=0, \\
\frac{\partial \vec{h}}{\partial t}=(\vec{H} \cdot \nabla) \vec{q}+\eta \nabla^{2} \vec{h} .
\end{gathered}
$$

Within the framework of Boussinesq approximation, equations (9) - (13), become

$$
\begin{aligned}
& \frac{\partial}{\partial t} \nabla^{2} w=\left(v+v^{\prime} \frac{\partial}{\partial t}\right) \nabla^{4} w+\frac{\mu_{e} H}{4 \pi \rho_{0}} \nabla^{2}\left(\frac{\partial h_{z}}{\partial z}\right)+g \alpha\left(\frac{\partial^{2} \theta}{\partial x^{2}}+\frac{\partial^{2} \theta}{\partial y^{2}}\right) \\
& \frac{\partial \theta}{\partial t}=\beta w+\kappa \nabla^{2} \theta \\
& \frac{\partial h_{z}}{\partial t}=H \frac{\partial w}{\partial z}+\eta \nabla^{2} h_{z} \\
& \text { where } \quad \nabla^{2}=\frac{\partial^{2}}{\partial x^{2}}+\frac{\partial^{2}}{\partial y^{2}}+\frac{\partial^{2}}{\partial z^{2}}
\end{aligned}
$$




\section{Normal Mode Analysis}

Analyzing the disturbances into normal modes, we assume that the Perturbation quantities are of the form

$$
\left\lfloor w, \theta, h_{z,}\right\rfloor=[W(z), \Theta(z), K(z)] \operatorname{Exp}\left(i k_{x} x+i k_{y} y+n t\right),
$$

Where $k_{x}, k_{y}$ are the wave numbers along the $\mathrm{x}$ - and $\mathrm{y}$-directions, respectively, $k=\left(k_{x}{ }^{2}+k_{y}{ }^{2}\right)^{\frac{1}{2}}$, is the resultant wave number, and $\mathrm{n}$ is the growth rate which is, in general, a complex constant.

Using (17), equations (14) - (16), in non-dimensional form transform to

$$
\begin{aligned}
& \left.\left(D^{2}-a^{2}\right)(1+F \sigma)\left(D^{2}-a^{2}\right)-\sigma\right\rfloor W=R a^{2} \Theta-Q\left(D^{2}-a^{2}\right) D K, \\
& \left(D^{2}-a^{2}-p_{1} \sigma\right) \Theta=-W,
\end{aligned}
$$

And

$\left(D^{2}-a^{2}-p_{2} \sigma\right) K=-D W$

Where we have introduced new coordinates $\left(x^{\prime}, y^{\prime}, z^{\prime}\right)=(\mathrm{x} / \mathrm{d}, \mathrm{y} / \mathrm{d}, \mathrm{z} / \mathrm{d})$ in new units of length $\mathrm{d}$ and $D=d / d z^{\prime}$. For convenience, the dashes are dropped hereafter. Also we have substituted $a=k d, \sigma=\frac{n d^{2}}{v}, p_{1}=\frac{v}{\kappa}$, is the thermal Prandtl number; $p_{2}=\frac{v}{\eta}$, is the magnetic Prandtl number; $F=\frac{v^{\prime}}{d^{2}}$, is the Rilvin-Ericksen kinematic viscoelasticity parameter; $R=\frac{g \alpha \beta d^{4}}{\kappa v}$, is the thermal Rayleigh number and $Q=\frac{\mu_{e} H^{2} d^{2}}{4 \pi \rho_{0} v \eta}$, is the Chandrasekhar number. Also we have Substituted $W=W_{\oplus}$, $\Theta=\frac{\beta d^{2}}{\kappa} \Theta_{\oplus}, K=\frac{H d}{\eta} K_{\oplus}$, and $D_{\oplus}=d D$, and dropped $(\oplus)$ for convenience.

We now consider the cases where the boundaries are rigid-rigid or rigid-free or free-rigid or free-free at $\mathrm{z}=$ 0 and $\mathrm{z}=1$, as the case may be, and are perfectly conducting. The boundaries are maintained at constant temperature, thus the perturbations in the temperature are zero at the boundaries. The appropriate boundary conditions with respect to which equations (18) -- (20), must possess a solution are
$\mathrm{W}=0=\Theta$,
on both the horizontal boundaries,
$\mathrm{DW}=0$, on a rigid boundary,

$$
D^{2} W=0 \text {, }
$$$$
\text { on a dynamically free boundary, }
$$
$\mathrm{K}=0$, on both the boundaries as the regions outside the fluid are perfectly conducting,

Equations (18) -- (20), along with appropriate boundary conditions (21) - (24), poses an eigenvalue problem for $\sigma$ and we wish to Characterize $\sigma_{i}$ when $\sigma_{r} \geq 0$.

We first note that since $W, \Theta$ and $\mathrm{K}$ satisfy $W(0)=0=W(1)$ and $K(0)=0=K(1)$ in addition to satisfying to governing equations and hence we have from the Rayleigh-Ritz inequality [18]

$$
\int_{0}^{1}|D W|^{2} d z \geq \pi^{2} \int_{0}^{1}|W|^{2} d z \text { and } \int_{0}^{1}|D K|^{2} d z \geq \pi^{2} \int_{0}^{1}|K|^{2} d z,
$$

Further, for $W(0)=0=W(1)$ and $K(0)=0=K(1)$, Banerjee et al. [19] have shown that

$$
\int_{0}^{1}\left|D^{2} W\right|^{2} d z \geq \pi^{2} \int_{0}^{1}|D W|^{2} d z \text { and } \int_{0}^{1}\left|D^{2} K\right|^{2} d z \geq \pi^{2} \int_{0}^{1}|D K|^{2} d z,
$$

We prove the following lemma:

\section{Mathematical Analysis}

Lemma 1: For any arbitrary oscillatory perturbation, neutral or unstable

$$
\int_{0}^{1}\left\{|D K|^{2}+a^{2}|K|^{2}\right\} d z \leq \frac{\pi^{2}}{\left(2 \pi^{2}-1\right)} \int_{0}^{1}|D W|^{2} d z
$$


Proof: Multiplying equation (20) by $K^{*}$ (the complex conjugate of $K$ ), integrating by parts each term of the resulting equation on the left hand side for an appropriate number of times and making use of boundary conditions on $K$ namely $K(0)=0=K(1)$, it follows that

$$
\begin{gathered}
\int_{0}^{1}\left\{|D K|^{2}+a^{2}|K|^{2}\right\} d z+\sigma_{r} p_{2} \int_{0}^{1}|K|^{2} d z=\text { Real part of }\left\{\int_{0}^{1} K^{*} D W d z\right\} \leq\left|\int_{0}^{1} K^{*} D W d z\right| \leq \int_{0}^{1}\left|K^{*} D W\right| d z \\
\leq \int_{0}^{1}\left|K^{*}\right||D W| d z \leq \frac{1}{2} \int_{0}^{1}\left(|K|^{2}+|D W|^{2}\right) d z
\end{gathered}
$$

which gives that

$\int_{0}^{1}|D K|^{2} d z \leq \frac{1}{2} \int_{0}^{1}\left(|K|^{2}+|D W|^{2}\right) d z$

inequality (28) on utilizing (25), gives

$$
\int_{0}^{1}|K|^{2} d z \leq \frac{1}{\left(2 \pi^{2}-1\right)} \int_{0}^{1}|D W|^{2} d z
$$

Since $\sigma_{r} \geq 0$ and $p_{2}>0$, hence inequality (27) on utilizing (29), give

$$
\int_{0}^{1}\left(|D K|^{2}+a^{2}|K|^{2}\right) d z \leq \frac{\pi^{2}}{\left(2 \pi^{2}-1\right)} \int_{0}^{1}|D W|^{2} d z,
$$

This completes the proof of lemma.

We prove the following theorems:

Theorem 1: If R $>0, \mathrm{~F}>0, Q>0, \sigma_{r} \geq 0$ and $\sigma_{i} \neq 0$ then the necessary condition for the existence of nontrivial solution $(W, \Theta, K)$ of equations (18) - (20) and the boundary conditions (21), (24) and any combination of (22) and (23) is that

$$
\left.\frac{Q p_{2} \pi^{2}}{\left(2 \pi^{2}-1\right)\left(1+\pi^{2} F\right)}\right\rangle 1
$$

Proof: Multiplying equation (18) by $W^{*}$ (the complex conjugate of $\mathrm{W}$ ) throughout and integrating the resulting equation over the vertical range of $z$, we get

$$
(1+F \sigma) \int_{0}^{1} W^{*}\left(D^{2}-a^{2}\right)^{2} W d z-\sigma \int_{0}^{1} W^{*}\left(D^{2}-a^{2}\right) W d z=R a^{2} \int_{0}^{1} W^{*} \Theta d z-Q \int_{0}^{1} W^{*} D\left(D^{2}-a^{2}\right) K d z,
$$

Taking complex conjugate on both sides of equation (19), we get

$$
\left(D^{2}-a^{2}-p_{1} \sigma^{*}\right) \Theta^{*}=-W^{*} \text {, }
$$

Therefore, using (32), we get

$$
\int_{0}^{1} W^{*} \Theta d z=-\int_{0}^{1} \Theta\left(D^{2}-a^{2}-p_{1} \sigma^{*}\right) \Theta^{*} d z,
$$

Also taking complex conjugate on both sides of equation (20), we get

$\left[D^{2}-a^{2}-p_{2} \sigma^{*}\right] K^{*}=-D W^{*}$,

Therefore, using (34) and using boundary condition (21), we get

$$
\int_{0}^{1} W^{*} D\left(D^{2}-a^{2}\right) K d z=-\int_{0}^{1} D W^{*}\left(D^{2}-a^{2}\right) K d z=\int_{0}^{1} K\left(D^{2}-a^{2}\right)\left(D^{2}-a^{2}-p_{2} \sigma^{*}\right) K^{*} d z,
$$

Substituting (33) and (35) in the right hand side of equation (31), we get

$$
\begin{aligned}
& (1+F \sigma) \int_{0}^{1} W^{*}\left(D^{2}-a^{2}\right)^{2} W d z-\sigma \int_{0}^{1} W^{*}\left(D^{2}-a^{2}\right) W d z \\
= & -R a^{2} \int_{0}^{1} \Theta\left(D^{2}-a^{2}-p_{1} \sigma^{*}\right) \Theta^{*} d z-Q \int_{0}^{1} K\left(D^{2}-a^{2}\right)\left(D^{2}-a^{2}-p_{2} \sigma^{*}\right) K^{*} d z,
\end{aligned}
$$


Integrating the terms on both sides of equation (36) for an appropriate number of times by making use of the appropriate boundary conditions (21) - (24), we get

$$
\begin{aligned}
& (1+F \sigma) \int_{0}^{1}\left(\left|D^{2} W\right|^{2}+2 a^{2}|D W|^{2}+a^{4}|W|^{2}\right) d z+\sigma \int_{0}^{1}\left(|D W|^{2}+a^{2}|W|^{2}\right) d z \\
& =R a^{2} \int_{0}^{1}\left\{\left.D \Theta\right|^{2}+a^{2}|\Theta|^{2}\right\} d z+R a^{2} p_{1} \sigma^{*} \int_{0}^{1}|\Theta|^{2} d z \\
& -Q \int_{0}^{1}\left(\left|D^{2} K\right|^{2}+2 a^{2}|D K|^{2}+a^{4}|K|^{2}\right) d z-Q p_{2} \sigma^{*} \int_{0}^{1}\left(|D K|^{2}+a^{2}|K|^{2}\right) d z .
\end{aligned}
$$

And equating the imaginary parts on both side of Eq. (37), and cancelling $\sigma_{i}(\neq 0)$ throughout, we get

$$
F \int_{0}^{1}\left(\left|D^{2} W\right|^{2}+2 a^{2}|D W|^{2}+a^{4}|W|^{2}\right) d z+\int_{0}^{1}\left(|D W|^{2}+a^{2}|W|^{2}\right) d z=-R a^{2} p_{1} \int_{0}^{1}|\Theta|^{2} d z+Q p_{2} \int_{0}^{1}\left(|D K|^{2}+a^{2}|K|^{2}\right) d z
$$

Now R $>0$ and $Q\rangle 0$, utilizing the inequalities (26) and (30), the equation (38) gives,

$$
\left[\left(1+\pi^{2} F\right)-\frac{Q p_{2} \pi^{2}}{\left(2 \pi^{2}-1\right)}\right] \int_{0}^{1}|D W|^{2} d z+a^{2} \int_{0}^{1}\left(2|D W|^{2}+|W|^{2}+a^{2}|W|^{2}\right) d z+R a^{2} p_{1} \int_{0}^{1}|\Theta|^{2} d z\langle 0,
$$

and therefore, we must have

$$
\left.\frac{Q p_{2} \pi^{2}}{\left(2 \pi^{2}-1\right)\left(1+\pi^{2} F\right)}\right\rangle 1
$$

Hence, if

$$
\left.\sigma_{r} \geq 0 \text { and } \sigma_{i} \neq 0, \text { then } \frac{Q p_{2} \pi^{2}}{\left(2 \pi^{2}-1\right)\left(1+\pi^{2} F\right)}\right\rangle 1 .
$$

And this completes the proof of the theorem.

Presented otherwise from the point of view of existence of instability as stationary convection, the above theorem can be put in the form as follow:-

Theorem 2: The sufficient condition for the validity of the 'exchange principle' and the onset of instability as a non-oscillatory motions of non-growing amplitude in a Rivlin-Ericksen viscoelastic fluid heated from below, in the presence of uniform vertical magnetic field is that $\frac{Q p_{2} \pi^{2}}{\left(2 \pi^{2}-1\right)\left(1+\pi^{2} F\right)} \leq 1$, where $Q$ is the Chandrasekhar number, $\mathrm{F}$ is the viscoelasticity parameter and $p_{2}$ the magnetic Prandtl number, for any combination of perfectly conducting dynamically free and rigid boundaries.

or

The onset of instability in Rivlin-Ericksen viscoelastic fluid heated from below, in the presence of uniform vertical magnetic field, cannot manifest itself as oscillatory motions of growing amplitude if the Chandrasekhar number $Q$, the viscoelasticity parameter $\mathrm{F}$ and the magnetic Prandtl number $p_{2}$, satisfy the inequality $\frac{Q p_{2} \pi^{2}}{\left(2 \pi^{2}-1\right)\left(1+\pi^{2} F\right)} \leq 1$, for any combination of perfectly conducting dynamically free and rigid boundaries.

In the context of existence of instability in 'oscillatory modes' and that of 'overstability in the present configuration, we can state the above theorem as follow:-

Theorem 3: The necessary condition for the existence of instability in 'oscillatory modes' and that of 'overstability' in a Rivlin-Ericksen viscoelastic fluid heated from below, in the presence of uniform vertical magnetic field, is that the Chandrasekhar number $Q$, the viscoelasticity parameter F and the magnetic Prandtl number $p_{2}$, must satisfy the inequality $\left.\frac{Q p_{2} \pi^{2}}{\left(2 \pi^{2}-1\right)\left(1+\pi^{2} F\right)}\right\rangle 1$, for any combination of perfectly conducting dynamically free and rigid boundaries. 


\section{Conclusions}

This theorem mathematically established that the onset of instability in a Rivlin-Ericksen viscoelastic fluid in the presence of uniform vertical magnetic field, cannot manifest itself as oscillatory motions of growing amplitude if the Chandrasekhar number $Q, \mathrm{~F}$ is the viscoelasticity parameter and $p_{2}$ the magnetic Prandtl number, satisfy the inequality $\frac{Q p_{2} \pi^{2}}{\left(2 \pi^{2}-1\right)\left(1+\pi^{2} F\right)} \leq 1$, for any combination of perfectly conducting dynamically free and rigid boundaries.

The essential content of the theorem, from the point of view of linear stability theory is that for the configuration of Rivlin-Ericksen viscoelastic fluid of infinite horizontal extension heated form below, having any combination of top and bottom horizontal bounding surfaces as free-free or free-rigid or rigid-free or rigid-rigid, and the region outside is perfectly conducting, in the presence of uniform vertical magnetic field parallel to the force field of gravity, an arbitrary neutral or unstable modes of the system are definitely non-oscillatory in character if $\frac{Q p_{2} \pi^{2}}{\left(2 \pi^{2}-1\right)\left(1+\pi^{2} F\right)} \leq 1$, and in particular PES is valid.

\section{References}

[1] H. Bénard, Les tourbillions cellulaires dans une nappe liquid, Revue Genérale des Sciences Pures et Appliquees 11 (1900), 1261$1271,1309-1328$.

[2] L. Rayleigh, On convective currents in a horizontal layer of fluid when the higher temperature is on the underside, Philosophical Magazine 32 (1916), 529-546.

[3] H. Jeffreys, The stability of a fluid layer heated from below, Philosophical Magazine 2 (1926), 833-844.

[4] S. Chandrasekhar, Hydrodynamic and Hydromagnetic Stability, Dover Publication, New York (1981).

[5] J.G. Oldroyd, Non-Newtonian effects in steady motion of some idealized elastic-viscous Liquids, Proceedings of the Royal Society of London A245 (1958), 278-297.

[6] R.S. Rivlin andJ.L. Ericksen, Stress deformation relations for isotropic materials, J. Rat. Mech. Anal.,4 (1955), 323.

[7] P.K. Bhatia and J.M. Steiner, Convective instability in a rotating viscoelastic fluid layer, Zeitschrift fur Angewandte Mathematik and Mechanik 52 (1972), 321-327.

[8] P.K. Bhatia and J.M. Steiner, Thermal Instability in a viscoelastic fluid layer in hydromagnetics, Journal of Mathematical Analysis and Applications 41 (1973), no. 2,271-283.

[9] R.C. Sharma, Effect of rotation on thermal instability of a viscoelastic fluid, Acta Physica Hungarica 40 (1976), 11-17.

[10] R.C. Sharma Thermal instability in a viscoelastic fluid in hydromagnetics, Acta Physica Hungarica 38 (1975), 293-298.

[11] R.C. Sharma and P. Kumar, Effect of rotation on thermal instability in Rivlin-Ericksen elastico-viscous fluid, Zeitschrift fur Naturforschung 51a (1996), 821-824.

[12] P. Kumar, H. Mohan and R. Lal, Effect of magnetic field on thermal instability of a rotating Rivlin-Ericksen viscoelastic fluid, Int. J. of Maths. Math. Scs., (2006), 1-10.

[13] A. Pellow and R.V. Southwell, R. On the maintained convective motion in a fluid heated from below. Proc. Roy. Soc. London A, (1940), 176, 312-43.

[14] M.B. Banerjee D.C. Katoch, G.S. Dube and K.Banerjee, Bounds for growth rate of perturbation in thermohaline convection. Proc. R. Soc. A, (1981), 378, 301-304

[15] M.B. Banerjee and B. Banerjee, A characterization of non-oscillatory motions in magnetohydronamics. Ind. J. Pure \& Appl Maths., (1984), 15(4), 377-382

[16] J.R. Gupta, S.K. Sood and U.D. Bhardwaj, On the characterization of nonoscillatory motions in rotatory hydromagnetic thermohaline convection, Indian J. pure appl.Math. 17(1), (1986), 100-107.

[17] A.S. Banyal, The necessary condition for the onset of stationary convection in couple-stress fluid, Int. J. of Fluid Mech. Research, 38(5), (2011), 450-457.

[18] M.H. Schultz, Spline Analysis, Prentice Hall, Englewood Cliffs, New Jersy9 (1973).

[19] M.B. Banerjee, J.R. Gupta and J. Prakash, On thermohaline convection of Veronis type, J. Math. Anal. Appl., Vol.179 (1992), pp. 327-334. 